\title{
List of Reviewers 2019
}

Thanks to our reviewers and corresponding editors!

The Journal of Pediatric Rehabilitation Medicine would like to recognize the following reviewers for their time and commitment in providing scientific peer reviews from 29-10-2018 to 4-11-2019.

\author{
Rishi Agrawal \\ Michele Alaniz \\ Andrew Althouse \\ Sandra Amaral \\ Lusine Ambartsumyan \\ Antonio Andreacchio \\ Morag Andrew \\ Rachel Annunziato \\ Frieda Ansoanuur \\ Joan Arvedson \\ manda Ayala \\ Andrea Bauer \\ Edward Bedrick \\ Patricia Beierwaltes \\ Melanie Bell \\ Leslie Benson \\ Patrizia Bertolini \\ Catherine Billups \\ Rhonda Bolin \\ Glendaliz Bosques \\ Elisabet Bousquet \\ Marina Brandão \\ Timothy Brei \\ Amy Brown \\ Cathleen Buckon \\ Anne Cain-Neilsen \\ Kristen Campbell \\ Jonathan Castillo \\ Yuping Chen \\ Yu-chun Chen \\ Earl Cheng \\ Wendy Chouteau \\ Laura Cif \\ Douglass Clayton \\ Gerald Clayton \\ Sean Cleary \\ John Connolly \\ Roger Cornwall \\ Robin Cotton
}

\author{
Bethany Coyne \\ Diane Damiano \\ Loren Davidson \\ Thomas Davis \\ Kathryn Davitt \\ Shay Dawson \\ Steven Day \\ Marisa De Cássia Registro Fonseca \\ Stephanie DeLuca \\ Christine Detrembleur \\ Cynthia Dodds \\ Rochelle Dy \\ Debbie Evans-Rogers \\ Nathan Evanson \\ Andrea Fergus \\ Janet Foote \\ Ellen Fremion \\ Deborah Gaebler-Spira \\ Mary Gannotti \\ Jay Goldsmith \\ Wendy Goodwin \\ Mark Gormley \\ Michael Green \\ Cameron Green \\ Yared Gurmu \\ Juliet Haarbauer-Krupa \\ Gang Han \\ Deanna Hansen \\ Heather Hardin \\ Paul Harmatz \\ Karen Harpster \\ Jill Heathcock \\ Gregory Heuer \\ Mary Jo Hidecker \\ Scott Hoffinger \\ Abigail Johnson \\ Harrison Jones \\ David Joseph \\ Denise Justice
}


John Kairalla

Sandra Kaplan

Natalie Karavarsamis

Elana Katz

Jaqueline Kauffman

J. King

Shelly King

Kat Kolaski

Marsha Lee

Esther Lee

Traci Leong

Nan Li

Chen Li

Yimei Li

Robert Lipschutz

Wei Liu

Doug Lorenz

Donovan Lott

Joyce Maring

Larissa Martins Garcia

Nicola Massy-Westropp

Janette McDougall

Jill Meilahn

Renato Melo

Elizabeth Moberg-Wolff

Heather Morris

Shubhra Mukherjee

Brooke Mulrenin

Erin Naber

Farideh Nejat

Virginia Nelson

Gary Nelson

Samuel Nemanich

Michael Nigro

Marykay Nutini

Kevin O'Brien

James Oglivie

Joyce Oleszek

Sharon O'Neil

Pornsuree Onmanee Kuvijitsuwan

Marisa Osorio

\author{
Stephen Parry \\ Peter Patrick \\ Moya Peterson \\ Frank Pidcock \\ Niveditta Ramkumar \\ Moya Monique Ridosh \\ Andrew Ries \\ Elias Rizk \\ Brandon Rocque \\ Dawn Roller \\ Phyllis Rowland \\ Peter Rumney \\ Suzanne Rybczynski \\ Sandra Saavedra \\ Jose Salazar-Torres \\ Donna Scarborough \\ Diane Sellers \\ Parag Shah \\ Susan Sienko \\ Hubert Swana \\ Kristen Taylor \\ Ryan Theis \\ Jeffrey Thomson \\ Deborah Thorpe \\ Rickey Tivis \\ Anja Van Campenhout \\ Alex Van Speybroeck \\ Laura Vogtle \\ Joshua Vova \\ Luke Wakely \\ Christa Walgaard \\ Kate Wan-Chu Chang \\ Binhuan Wang \\ Seth Warschausky \\ Ashley Whitaker \\ Sian Williams \\ Colleen Wunderlich \\ Joseph (Zhe) Xu \\ Michele Zaccario \\ Hui Zhang \\ Alexander Ozonoff
}

JPRM would also like to thank our corresponding editors during this time:

Simra Javaid

Katy Ruch

Mi Ran Shin

We would also like to thank the Foundation for P M \& R for their generous support this year. 